\title{
Anharmonic Origin of the Giant Thermal Expansion of $\mathrm{NaBr}$
}

\author{
Y. Shen $\odot,{ }^{1, *}$ C. N. Saunders $\odot,{ }^{1}$ C. M. Bernal $\odot,{ }^{1}$ D. L. Abernathy $\odot,{ }^{2}$ M. E. Manley $\odot{ }^{3}$ and B. Fultz $\oplus^{1, \dagger}$ \\ ${ }^{1}$ Department of Applied Physics and Materials Science, California Institute of Technology, Pasadena, California 91125, USA \\ ${ }^{2}$ Neutron Scattering Division, Oak Ridge National Laboratory, Oak Ridge, Tennessee 37831, USA \\ ${ }^{3}$ Materials Science and Technology Division, Oak Ridge National Laboratory, Oak Ridge, Tennessee 37831, USA
}

(Received 31 August 2019; revised 9 June 2020; accepted 24 July 2020; published 21 August 2020)

All phonons in a single crystal of $\mathrm{NaBr}$ are measured by inelastic neutron scattering at temperatures of 10, 300, and $700 \mathrm{~K}$. Even at $300 \mathrm{~K}$, the phonons, especially the longitudinal-optical phonons, show large shifts in frequencies and show large broadenings in energy owing to anharmonicity. A $b$ initio computations are first performed with the quasiharmonic approximation (QHA) in which the phonon frequencies depend only on $V$ and on $T$ only insofar as it alters $V$ by thermal expansion. This QHA is an unqualified failure for predicting the temperature dependence of phonon frequencies, even $300 \mathrm{~K}$, and the thermal expansion is in error by a factor of 4 . Ab initio computations that include both anharmonicity and quasiharmonicity successfully predict both the temperature dependence of phonons and the large thermal expansion of $\mathrm{NaBr}$. The frequencies of longitudinal-optical phonon modes decrease significantly with temperature owing to the real part of the phonon self-energy from explicit anharmonicity originating from the cubic anharmonicity of nearest-neighbor $\mathrm{NaBr}$ bonds. Anharmonicity is not a correction to the QHA predictions of thermal expansion and thermal phonon shifts but dominates the behavior.

DOI: 10.1103/PhysRevLett.125.085504

Thermal expansion, a fundamental thermophysical property, originates primarily from a competition between the elastic energy of expansion and the phonon entropy, which usually increases beyond harmonic behavior as a solid expands. Thermal expansion can be calculated readily in the quasiharmonic approximation (QHA), which assumes that phonon frequencies depend only on volume [1-6]. The QHA theory of thermal expansion is textbook content and is logically self-consistent. It ignores explicit anharmonicity, where phonon frequencies also change with temperature at a fixed volume [7-9]. Some calculations include anharmonicity as a small correction to the QHA, but the relative importance of anharmonicity is not yet settled $[2,10]$.

We recently found that the QHA gave the wrong sign for the temperature dependence of most phonons in silicon [11]. This shows that the QHA is physically incomplete, even though it did predict the thermal expansion correctly. Here we report a more compelling inelastic neutron scattering (INS) experiment to test predictions of phonons and thermal expansion in a different material, sodium bromide $(\mathrm{NaBr})$. Like other alkali halides with the rocksalt structure [12-14], $\mathrm{NaBr}$ has received special attention owing to its cubic structure and highly ionic bonding.

The INS data from a single crystal of $\mathrm{NaBr}$ were acquired with the time-of-flight spectrometer ARCS [15] at the Spallation Neutron Source at the Oak Ridge National Laboratory, using neutrons with an incident energy of $30 \mathrm{meV}$. Data were collected from 201 rotations of the crystal in increments of $0.5^{\circ}$ about the vertical [001] axis. Data reduction gave the $4 \mathrm{D}$ scattering function $S(\mathbf{Q}, \varepsilon)$
$[16,17]$, where $\mathbf{Q}$ is the 3D wave vector and $\varepsilon$ is the phonon energy (from the neutron energy loss). Nonlinearities of the ARCS instrument were corrected with a small linear rescaling of the $q$ grid, calibrated by the positions of 45 in situ Bragg diffractions. After subtracting the background from measurements on an empty can at the same temperature and removing multiphonon scattering with the incoherent approximation, the higher Brillouin zones were folded back into an irreducible wedge in the first Brillouin zone to obtain the spectral intensities shown in Fig. 1. The Supplemental Material [18] describes the experiment and data analysis in more detail.

The QHA uses an explicit dependence of phonon frequencies on volume into the Helmholtz free energy

$$
\begin{aligned}
F^{\mathrm{QHA}}(T, V)= & U_{0}(V)+\sum_{\mathbf{q}, j}\left[\frac{\hbar \omega_{\mathbf{q}, j}(V)}{2}\right. \\
& \left.+k_{B} T \ln \left(1-\exp \left\{-\frac{\hbar \omega_{\mathbf{q}, j}(V)}{k_{B} T}\right\}\right)\right],
\end{aligned}
$$

where $U_{0}(V)$ is the ground-state internal energy without any vibrational contribution and the term $k_{B} \ln [\ldots]$ includes the entropy that depends on volume through the individual phonon frequencies $\omega_{\mathbf{q}, j}=\omega_{\mathbf{q}, j}(V)$ (for the $j$ th phonon branch at wave vector $\mathbf{q}$ ). The finite-displacement method, as implemented in Phonopy [19], was used to obtain phonon frequencies for different volumes by density functional theory calculations with the Vienna ab initio simulation package [20-23]. The equilibrium volume at a given temperature $T$ was obtained by minimizing $F^{\mathrm{QHA}}(T, V)$ 


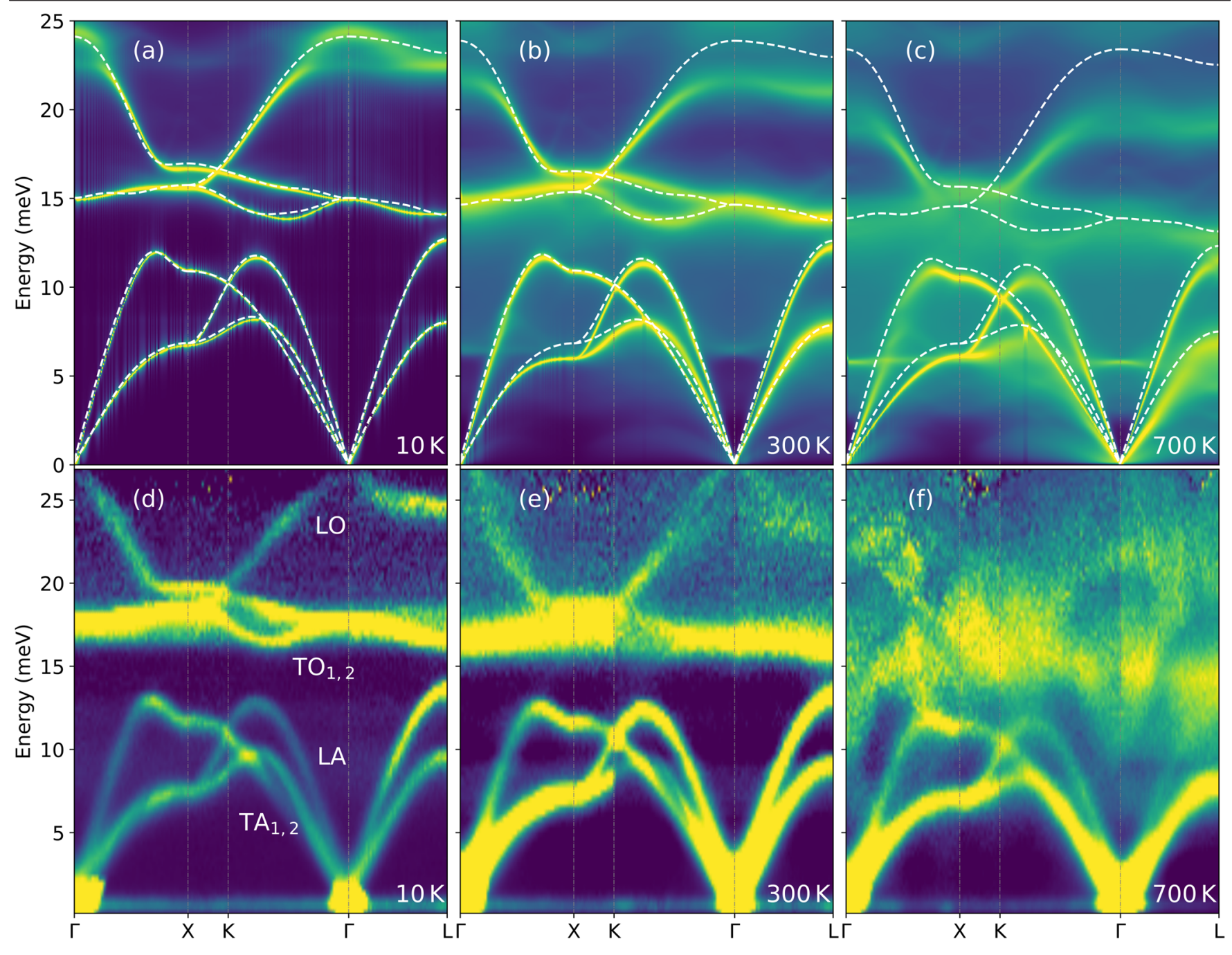

FIG. 1. Comparison between computational (QHA and fully anharmonic) and experimental (INS) results on phonon dispersions of $\mathrm{NaBr}$. (a)-(c) Phonons in $\mathrm{NaBr}$ calculated with the QHA (white dotted line) and from the full phonon spectral function (logarithmic intensity map) from sTDEP. Temperatures are labeled in the panels. (d)-(f) Corresponding 2D slices through the 4D scattering function $S(\mathbf{Q}, \varepsilon)$ from INS, where $\varepsilon=\hbar \omega$, along high symmetry lines in the first Brillouin zone.

with respect to volume $V$, keeping $T$ as a fixed parameter. Figure 2 shows how the QHA fails to predict both the magnitude and shape of the thermal expansion curve of $\mathrm{NaBr}$, even at room temperature.

Anharmonic behavior was calculated by the stochastically initialized temperature dependent effective potential method (sTDEP) [24-26,42]. In sTDEP, the BornOppenheimer molecular dynamics potential energy surface of $\mathrm{NaBr}$ was evaluated by a Monte Carlo sampling of the phase space of atom positions. The forces on atoms were fitted to a model Hamiltonian,

$$
\begin{aligned}
\hat{H}= & U_{0}+\sum_{i} \frac{\mathbf{p}_{i}^{2}}{2 m_{i}}+\frac{1}{2 !} \sum_{i j} \sum_{\alpha \beta} \Phi_{i j}^{\alpha \beta} u_{i}^{\alpha} u_{j}^{\beta} \\
& +\frac{1}{3 !} \sum_{i j k} \sum_{\alpha \beta \gamma} \Phi_{i j k}^{\alpha \beta \gamma} u_{i}^{\alpha} u_{j}^{\beta} u_{k}^{\gamma},
\end{aligned}
$$

by density functional theory calculations on various configurations of displaced atoms by stochastic sampling of a canonical ensemble, with Cartesian displacements $\left(u_{i}^{\alpha}\right)$ normally distributed around the mean thermal displacement. The $U_{0}$ is a fit parameter for the baseline of the potential energy surface. The temperature-dependent $\left\{\Phi_{i j}\right\}$ were used to calculate phonon frequencies. The cubic force constants $\Phi_{i j k}$ capture the broadening and additional shifts of phonon modes, as discussed below.

The vibrational free energies on a $V$ - $T$ grid were directly calculated from the anharmonic phonon DOS by the sTDEP method. The Helmholtz free energy $F(V, T)$ of Eq. (1) was calculated with $\omega_{\mathbf{q}, j}^{\mathrm{anh}}$ instead of the quasiharmonic $\omega_{\mathbf{q}, j}$, thereby including the anharmonic phonon effects explicitly in the same free energy used to predict the thermal expansion. The equilibrium volumes were obtained by minimization of the Helmholtz free energy at $T$, giving 


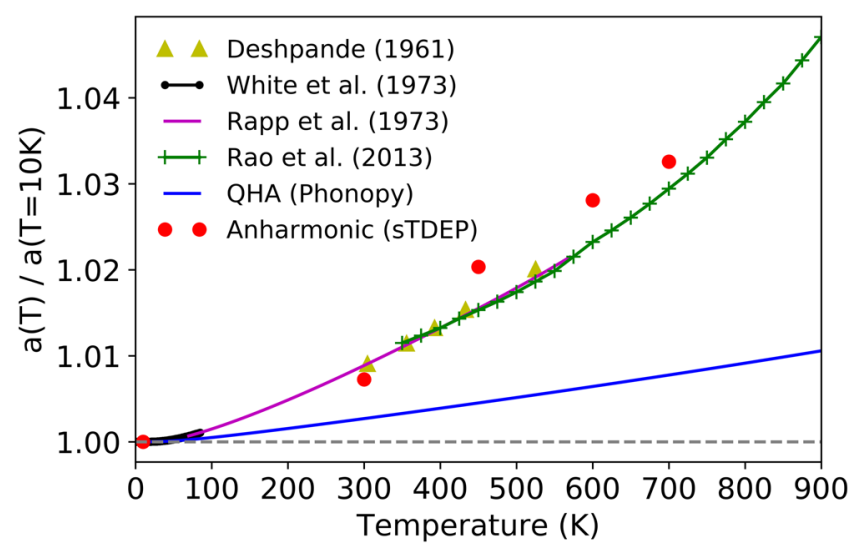

FIG. 2. Thermal expansion of $\mathrm{NaBr}$. The $a b$ initio QHA (blue solid line) and anharmonic calculations (red solid circles) are compared to experimental results $[27-29,43]$. There is a large discrepancy between the measurements and the QHA predictions, while results from the sTDEP method are in close agreement with the experiments.

the results shown in Fig. 2. These equilibrium volumes are in good agreement with experimental measurements, although there are deviations at higher temperatures. Details of the calculations of equilibrium volumes and phonon dispersions are given in the Supplemental Material [18].

Some calculated phonon spectral weights are compared to experimental measurements in Fig. 1 along directions of high symmetry. At $10 \mathrm{~K}$, all calculations agree with each other and with the experimental measurements. At higher temperatures, the acoustic dispersions below $14 \mathrm{meV}$ show some softening, especially at $700 \mathrm{~K}$, but are not broadened so much as the optical modes. The optical modes show large broadening at $300 \mathrm{~K}$ and major changes in shape at $700 \mathrm{~K}$. The temperature dependence of the optical dispersions is largely captured by the spectral weight calculated by sTDEP, but only a minor part of the softening is predicted by the QHA calculations (and none of the broadening owing to its assumption of noninteracting modes). The largest contribution to the temperature shift of the spectral weight is from the real part of the cubic term, obtained as a Kramers-Kronig transformation of the imaginary part of the self-energy as explained in the Supplemental Material [18] with Eq. (51). The imaginary part of the phonon self-energy from this cubic term is responsible for the surprisingly large energy broadening of the longitudinal-optical (LO) phonons at $300 \mathrm{~K}$ and especially at $700 \mathrm{~K}$.

The experimental INS measurements (see Fig. 1) were fitted to give the energy shifts of LO phonons presented in Table I. The QHA accounts for only a small part of the experimental shifts, but the anharmonic calculations are much more successful. The spectral intensities at the $L$ point are shown in Fig. 3(a). All phonons at the $L$ point soften and broaden significantly with temperature. Spectra from the longitudinal-acoustic and transverse-optic phonon modes merge into one broad peak at $700 \mathrm{~K}$. The LO peak broadens significantly, but its large thermal softening is still evident. Figure 3(b) shows that the real part of the selfenergy of the LO phonon at the $L$ point is approximately $-3.5 \mathrm{meV}$ at $700 \mathrm{~K}$, so phonon-phonon anharmonicity dominates the thermal shift of this mode (the LO mode has a phonon energy of $19 \mathrm{meV}$ at the $L$ point from sTDEP). The Supplemental Material [18] shows some of the spectral weights in more detail. There are differences between experiment and the sTDEP calculations at $700 \mathrm{~K}$, especially halfway between $\Gamma$ and $L$ between 16 and $23 \mathrm{meV}$. Some anharmonic effects in $\mathrm{NaBr}$ are too large to be predicted accurately by the sTDEP method.

To understand the origin of the anharmonicity at $700 \mathrm{~K}$, the cubic irreducible force constants for the three-body interactions within the first ten coordination shells were individually set to zero while recalculating phonon line shapes at different $\mathbf{Q}$. Figure 3(c) shows how two related irreducible force constants dominate the line shapes. They correspond to the nearest-neighbor cubic interactions of degenerate triplets $(\mathrm{NaNaBr}$ and/or $\mathrm{NaBrBr})$ in the [100] direction (i.e., along the $\mathrm{NaBr}$ bond direction). (By translational invariance, $\Phi_{\mathrm{NaNaBr}}^{\alpha \alpha \alpha}=-\Phi_{\mathrm{NaBrBr}}^{\alpha \alpha \alpha}$.) When these force constants are switched off, the phonon line shapes revert to narrow Lorentzian functions typical of weakly anharmonic solids, and these Lorentzian peaks are at energies similar to those from the QHA calculations. The dominance of $\Phi_{\mathrm{NaNaBr}}^{\alpha \alpha \alpha}=-\Phi_{\mathrm{NaBrBr}}^{\alpha \alpha \alpha}$ on the phonon anharmonicity was found for phonons at all other points in reciprocal space, as shown in the Supplemental Material [18].

TABLE I. Phonon energy shifts of the LO mode with temperature.

\begin{tabular}{|c|c|c|c|c|c|c|c|c|c|}
\hline \multirow[b]{3}{*}{$T(\mathrm{~K})$} & \multicolumn{9}{|c|}{ Energy shift: $\left(\varepsilon-\varepsilon_{10 \mathrm{~K}}\right) / \varepsilon_{10 \mathrm{~K}}$} \\
\hline & \multicolumn{3}{|c|}{ At $L$ point } & \multicolumn{3}{|c|}{ Along $\Gamma-L$} & \multicolumn{3}{|c|}{ Along $\Gamma-X$} \\
\hline & $\mathrm{QH}$ & $\mathrm{ANH}$ & EXP & $\mathrm{QH}$ & $\mathrm{ANH}$ & EXP & $\mathrm{QH}$ & $\mathrm{ANH}$ & EXP \\
\hline 300 & -0.003 & -0.065 & $-0.080(0.020)$ & -0.037 & -0.087 & $-0.062(0.020)$ & -0.031 & -0.051 & $-0.052(0.020)$ \\
\hline 700 & -0.025 & -0.164 & $-0.174(0.055)$ & -0.045 & -0.181 & $-0.169(0.055)$ & -0.034 & -0.144 & $-0.132(0.055)$ \\
\hline
\end{tabular}

$\mathrm{QH}=$ quasiharmonic, $\mathrm{ANH}=$ anharmonic, $\mathrm{EXP}=$ experimental.

Errors are from the instrument energy resolution and/or the peak fitting process.

Average values were used for evaluation along the path. 

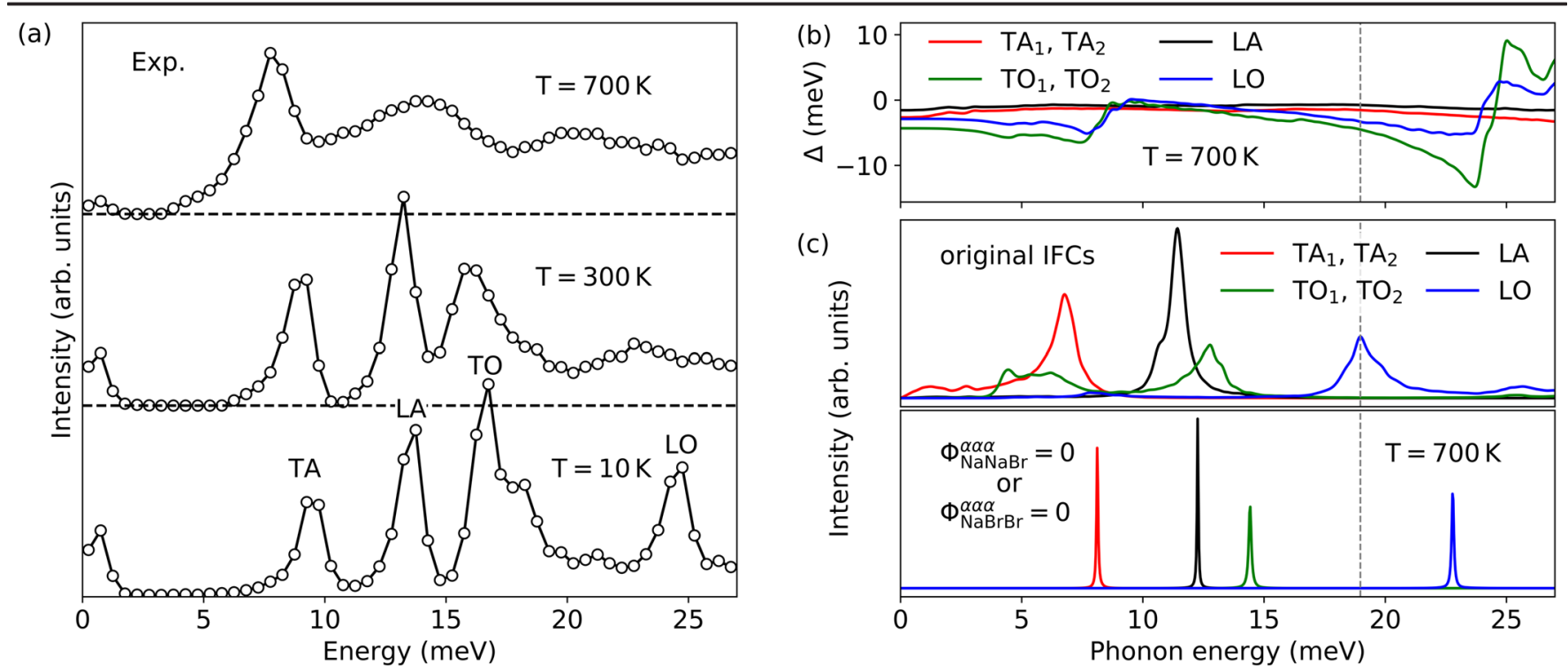

FIG. 3. Measured and calculated phonon line shapes at the $L$ point and the real part of the phonon self-energy. (a) The 1D cut of $S(\mathbf{Q}, \varepsilon)$ at a constant $\mathbf{Q}=[0.5,0.5,0.5]$ r.l.u. (reciprocal lattice units) showing the temperature dependence of phonon line shapes. (The small peak near zero is the residue from elastic scattering after correcting for the phonon creation thermal factor.) (b) Real component of the phonon self-energy $\Delta$ from the third-order force constants. (c) Phonon intensities after nulling the third-order force constants, $\Phi_{\mathrm{NaNaBr}}^{\alpha \alpha \alpha}$ or $\Phi_{\mathrm{NaBrBr}}^{\alpha \alpha \alpha}$ associated with the nearest-neighbor degenerate triplets, where $\alpha=(x, y, z)$ represents the direction along the $\mathrm{NaBr}$ bond.

The physics of thermal expansion requires volume and temperature derivatives of $F(V, T)$, specifically $\partial^{2} F /(\partial V \partial T)=-\beta B_{T}$. The Supplemental Material [18] obtains an expression for the ratio between $\beta^{\mathrm{QH}}$, the thermal expansion in the QHA, and the real $\beta$. For $\hbar \omega_{\max }<k_{B} T$,

$\beta^{\mathrm{QH}} / \beta \simeq 1-\frac{6 k_{B}}{B_{T} \beta v}\left(\bar{\gamma}_{V, T}+\bar{\gamma}_{V} \bar{\gamma}_{T}\right) \simeq 1-\frac{6 k_{B}}{B_{T} \beta v} \bar{\gamma}_{V, T}$,

where $B_{T}$ is the isothermal bulk modulus, $v$ is the volume per atom, the mode Grüneisen parameter is $\gamma_{V} \triangleq$ $-\left.(V / \omega)(\partial \omega / \partial V)\right|_{T}$, the thermal Grüneisen parameter is $\gamma_{T} \triangleq-\left.(T / \omega)(\partial \omega / \partial T)\right|_{V}$, the mode anharmonicity parameter is

$$
\gamma_{V, T} \triangleq-\frac{V T}{\omega}\left(\frac{\partial^{2} \omega}{\partial T \partial V}\right)
$$

and $\bar{\gamma}_{V, T}$ is the average anharmonicity parameter. For $\mathrm{NaBr}$, $\beta^{\mathrm{QH}} \simeq 0.35 \beta$, which is consistent with Fig. 2 above.

By testing different first principles calculations against phonons measured by inelastic neutron scattering at different temperatures, we demonstrated that the widely accepted quasiharmonic method predicts only a small fraction of the thermal phonon shifts and the thermal expansion. Anharmonic effects drastically alter the phonon self-energies, especially the LO phonons. The dominant anharmonicity is from cubic interactions associated with the nearest-neighbor degenerate triplets along the $\mathrm{NaBr}$ bonding direction. The volume dependence of the phonon anharmonicity dominates the thermal expansion of $\mathrm{NaBr}$.
Other alkali halides with the rocksalt structure, especially $\mathrm{NaCl}, \mathrm{NaI}$, and $\mathrm{NaF}$, have similar thermal expansion as $\mathrm{NaBr}$ and similar Grüneisen parameters of 3.3 when calculated as $\gamma=\beta B v / C_{p}$. Similar anharmonic effects seem likely in these compounds.

We thank D. Kim, O. Hellman, F. Yang, and J. Lin for helpful discussions. M.E. M. was supported by the U.S. Department of Energy (DOE), Office of Science, Office of Basic Energy Sciences, Materials Sciences, and Engineering Division under Award No. DE-AC05-00OR22725. Research with the Spallation Neutron Source at the Oak Ridge National Laboratory was sponsored by the Scientific User Facilities Division, Basic Energy Sciences, of the DOE. This work used resources from National Energy Research Scientific Computing Center, an Office of Science User Facility supported by the Office of Science of the US DOE under Award No. DE-AC02-05CH11231. This work was supported by the DOE Office of Science, Basic Energy Sciences, under Award No. DE-FG0203ER46055.

* Corresponding author. yshen@caltech.edu Corresponding author. btf@caltech.edu

[1] Z.-K. Liu, Y. Wang, and S. Shang, Sci. Rep. 4, 7043 (2015).

[2] P. B. Allen, Mod. Phys. Lett. B 34, 2050025 (2020).

[3] R. Mittal, M. Gupta, and S. Chaplot, Prog. Mater. Sci. 92, 360 (2018). 
[4] P. Nath, J. J. Plata, D. Usanmaz, R. A. R. A. Orabi, M. Fornari, M. B. Nardelli, C. Toher, and S. Curtarolo, Comput. Mater. Sci. 125, 82 (2016).

[5] G. Grimvall, Thermophysical Properties of Materials (Elsevier, North-Holland, 1999).

[6] A. A. Quong and A. Y. Liu, Phys. Rev. B 56, 7767 (1997).

[7] K. V. Zakharchenko, M. I. Katsnelson, and A. Fasolino, Phys. Rev. Lett. 102, 046808 (2009).

[8] C. W. Li, X. Tang, J. A. Muñoz, J. B. Keith, S. J. Tracy, D. L. Abernathy, and B. Fultz, Phys. Rev. Lett. 107, 195504 (2011).

[9] T. Lan, C. W. Li, J. L. Niedziela, H. Smith, D. L. Abernathy, G. R. Rossman, and B. Fultz, Phys. Rev. B 89, 054306 (2014).

[10] A. Erba, M. Shahrokhi, R. Moradian, and R. Dovesi, J. Chem. Phys. 142, 044114 (2015).

[11] D. S. Kim, O. Hellman, J. Herriman, H. L. Smith, J. Y. Y. Lin, N. Shulumba, J. L. Niedziela, C. W. Li, D. L. Abernathy, and B. Fultz, Proc. Natl. Acad. Sci. U.S.A. 115, 1992 (2018).

[12] V. Mitskevich, Sov. Phys. Solid State 3, 2202 (1962).

[13] E. Cowley and R. A. Cowley, Proc. R. Soc. 287, 259 (1965).

[14] R. Cowley, Rep. Prog. Phys. 31, 123 (1968).

[15] D. L. Abernathy, M. B. Stone, M. Loguillo, M. Lucas, O. Delaire, X. Tang, J. Lin, and B. Fultz, Rev. Sci. Instrum. 83, 015114 (2012).

[16] R. Ewings, A. Buts, M. Le, J. van Duijn, I. Bustinduy, and T. Perring, Nucl. Instrum. Methods Phys. Res., Sect. A 834, 132 (2016).

[17] C. W. Li, O. Hellman, J. Ma, A. F. May, H. B. Cao, X. Chen, A. D. Christianson, G. Ehlers, D. J. Singh, B. C. Sales, and O. Delaire, Phys. Rev. Lett. 112, 175501 (2014).

[18] See Supplemental Material at http://link.aps.org/ supplemental/10.1103/PhysRevLett.125.085504 for a detailed description of the calculations and experiments, which includes Refs. [2,19-41].

[19] A. Togo and I. Tanaka, Scr. Mater. 108, 1 (2015).

[20] G. Kresse and J. Hafner, Phys. Rev. B 47, 558 (1993).

[21] G. Kresse and J. Hafner, Phys. Rev. B 49, 14251 (1994).
[22] G. Kresse and J. Furthmüller, Comput. Mater. Sci. 6, 15 (1996).

[23] G. Kresse and J. Furthmüller, Phys. Rev. B 54, 11169 (1996).

[24] O. Hellman, I. A. Abrikosov, and S. I. Simak, Phys. Rev. B 84, 180301(R) (2011).

[25] O. Hellman, P. Steneteg, I. A. Abrikosov, and S. I. Simak, Phys. Rev. B 87, 104111 (2013).

[26] O. Hellman and I. A. Abrikosov, Phys. Rev. B 88, 144301 (2013).

[27] G. K. White, J. G. Collins, and K. A. G. Mendelssohn, Proc. R. Soc. A 333, 237 (1973).

[28] J. E. Rapp and H. D. Merchant, J. Appl. Phys. 44, 3919 (1973).

[29] A. S. M. Rao, K. Narender, K. G. K. Rao, and N. G. Krishna, J. Mod. Phys. 04, 208 (2013).

[30] D. C. Wallace, Statistical Physics of Crystals and Liquids (World Scientific, Singapore, 2002), p. 193.

[31] R. A. Cowley, Proc. Phys. Soc. 90, 1127 (1967).

[32] J. A. Reissland, The Physics of Phonons (John Wiley and Sons, New York, 1973).

[33] O. L. Anderson, Phys. Rev. 144, 553 (1966).

[34] Y. Sato-Sorensen, J. Geophys. Res. Solid Earth 88, 3543 (1983).

[35] V. F. Sears, Phys. Rev. A 7, 340 (1973).

[36] G. Kresse and D. Joubert, Phys. Rev. B 59, 1758 (1999).

[37] J. P. Perdew, K. Burke, and M. Ernzerhof, Phys. Rev. Lett. 77, 3865 (1996).

[38] M. Gajdoš, K. Hummer, G. Kresse, J. Furthmüller, and F. Bechstedt, Phys. Rev. B 73, 045112 (2006).

[39] X. Gonze and C. Lee, Phys. Rev. B 55, 10355 (1997).

[40] D.C. Wallace, Thermodynamics of Crystals (Wiley, New York, 1972).

[41] N. Shulumba, O. Hellman, and A. J. Minnich, Phys. Rev. B 95, 014302 (2017).

[42] V. Popescu and A. Zunger, Phys. Rev. B 85, 085201 (2012).

[43] V. Deshpande, Acta Crystallogr. 14, 794 (1961). 